\title{
Stage IVA Laryngeal Cancer AJCC v8
}

National Cancer Institute

\section{Source}

National Cancer Institute. Stage IVA Laryngeal Cancer AJCC v8. NCI Thesaurus. Code C133162.

Stage IVA includes: (T4a, N0, M0); (T4a, N1, M0); (T1, N2, M0); (T2, N2, M0); (T3, N2, M0); (T4a, N2, M0). T4a: Suprag lottis: Moderately advanced local disease. Tumor invades through the outer cortex of the thyroid cartilage and/or invades tissues beyond the larynx (e.g., trachea, soft tissues of neck including deep extrinsic muscle of the tongue, strap muscles, thyroid, or esophagus). Glottis: Moderately advanced local disease. Tumor invades through the outer cortex of the thyroid cartilage and/or invades tissues beyond the larynx (e.g., trachea, cricoid cartilage, soft tissues of neck including deep extrinsic muscle of the tongue, strap muscles, thyroid, or esophagus). Subglottis: Moderately advanced local disease. Tumor invades cricoid or thyroid cartilage and/or invades tissues beyond the larynx (e.g., trachea, soft tissues of neck including deep extrinsic muscles of the tongue, strap muscles, thyroid, or esophagus). T3: Supraglottis: Tumor is limited to larynx with vocal cord fixation and/or invades any of the following: postcricoid area, preepiglottic space, paraglottic space, and/or inner cortex of thyroid cartilage. Glottis: T umor is limited to the larynx with vocal cord fixation and /or invades the paraglottic space, and/or inner cortex of the thyroid cartilage. Subglottis: Tumor is limited to the larynx with vocal cord fixation and/or invades the paraglottic space and/or inner cortex of the thyroid cartilage. T1: Supraglottis: Tumor is limited to one subsite of suprag lottis with normal vocal cord mobility. Glottis: Tumor is limited to the vocal cord(s) (may involve anterior or posterior commissure) with normal mobility. Subglottis: Tumor is limited to the subglottis. T2: Supraglottis: Tumor invades the mucosa of more than one adjacent subsite of suprag lottis or glottis or region outside the suprag lottis (e.g., mucosa of base of the tongue, vallecula, medial wall of pyriform sinus) without fixation of the larynx. Glottis: Tumor extends to supraglottis and/or subglottis, and/or with impaired vocal cord mobility. Subglottis: T umor extends to vocal cord(s) with normal or impaired mobility. N0: No regional lymph node metastasis. N1: Metastasis in a single ipsilateral lymph node, $3 \mathrm{~cm}$ or less in greatest dimension and ENE(-). N2: Metastasis in a single ipsilateral lymph node, $3 \mathrm{~cm}$ or less in greatest dimension and $\mathrm{ENE}(+)$; or metastasis in a 
single ipsilateral lymph node, more than $3 \mathrm{~cm}$ but not more than $6 \mathrm{~cm}$ in greatest dimension and ENE(-); or metastases in multiple ipsilateral lymph nodes, none more than $6 \mathrm{~cm}$ in greatest dimension and $\mathrm{ENE}(-)$; or metastasis in bilateral or contralateral lymph nodes, none more than $6 \mathrm{~cm}$ in greatest dimension and ENE(-). M0: No distant metastasis. (AJCC 8th ed.) 